\section{Prädiktoren zur Stabilisierung opioidabhängiger gravider Frauen mit Ausblick auf die elterliche Sorge}

Romana Ortner ${ }^{1}$, Anna Schuster ${ }^{1}$, Claudia Rohrmeister ${ }^{2}$, Gabriele Fischer $^{1}$

${ }^{1}$ Drogenambulanz, Klinische Abteilung für Allgemeine Psychiatrie, Universitätsklinik für Psychiatrie, Wien

2 Klinische Abteilung für Neonatologie, Universitätsklinik für Kinderheilkunde, Wien
Zusammenfassung: Ziele: Es sollen verschiedene Faktoren aufgezeigt werden, die zu einer Stabilisierung der opioidabhängigen Graviden führen und damit die Voraussetzung für die Fürsorge ihrer Kinder schaffen. Methode: Die Patientinnen wurden ambulant in einem standardisierten Setting entweder mit Methadon, retardiertem Morphin oder Buprenorphin behandelt. Als Zielparameter für den Verbleib des Kindes wurden der Zeitpunkt des Behandlungsbeginnes, die Retentionsrate, die Dauer und Art der Opioiderhaltungstherapie sowie die Frequenz der Teilnahme an der psychotherapeutischen Gruppe evaluiert. Patientinnen: Während des Untersuchungszeitraums von 42 Monaten wurden 113 schwangere opioidabhängige Frauen eingeschlossen. Ergebnisse: Von 94 im Untersuchungszeitraum geborenen Kindern waren zum Nacherhebungszeitpunkt noch 59,6\% der Kinder $(n=56)$ in mütterlicher Sorge. Die Dauer der Opioiderhaltungstherapie ( $p=0,02)$ sowie die frühzeitige therapeutische Intervention $(p=0,03)$ und die Frequenz der Teilnahme an der Psychotherapie zeigten einen signifikanten Einfluss auf den Verbleib des Kindes. Diskussion: Substanzabhängigen graviden Frauen sollte ein frühzeitiger Behandlungsbeginn in einer multiprofessionellen Betreuungseinrichtung ermöglicht werden und dafür gilt es, Voraussetzungen zu schaffen.

Schlüsselwörter: Schwangerschaft - Opioide - Substanzabhängigkeit - Psychotherapie - Elterliche Sorge

Predictors for the Stabilization of Opioid Dependent Pregnant Women with an Outlook onto Parental Care: Aims: The aim of our study was to perform an analysis of different variables to determine which factors are responsible for stabilizing pregnant addicts, so that they could keep their child. Methods: All patients have been recruited through the drug outpatient clinic and had access to a standardized setting, including psychosocial and psychotherapeutical interventions. They were treated either with methadone, slow-release morphine or buprenorphine. Outcome measures whether the child can live with its own mother, were the time of enrolement, the length of treatment period, type and duration of maintenance therapy as well as attendance of psychotherapy. Patients: During the investigational period of 42 months 61 pregnant opioid (DSM IV: 304.0) and 52 polysubstance (DSM IV: 304.8) dependent pregnant

Suchttherapie 2001; 2: 152-158

(c) Georg Thieme Verlag Stuttgart · New York ISSN 1439-9903 women were enroled in the treatment program. Results: 94 children were born during the investigation period. At the end of this time frame $59.6 \%(n=56)$ of the children were still raised by their mothers. Duration of involvement in a maintenance program with replacement opioids $(p=0.02)$, as well as early intervention $(p=0.03)$ had a significant influence towards retaining custody of the child. In addition to successful pharmacological treatment, psychosocial and psychotherapeutical services influenced the outcome in respect of mothers keeping the child. Discussion: Special training programs of health care professionals could support early intervention and effective multiprofessional treatment that should be offered to each pregnant addicted woman.

Key words: Pregnancy - Opioids - Substance Dependence Psychotherapy - Custody of the Child

\section{Einleitung}

In den letzten Jahrzehnten hat die Auseinandersetzung mit dem Thema Sucht und Suchtverhalten einen zunehmend größeren Raum eingenommen und dennoch wurde dem Thema Substanzabhängigkeit und Schwangerschaft bislang nur ungenügend Aufmerksamkeit geschenkt [1], obwohl das Durchschnittsalter der Abhängigen von illegalen Substanzen bei Erstkontakt in Einrichtungen zwischen 20 und 30 Jahren liegt.

Gerade schwangere, opioidabhängige Patientinnen bedürfen einer besonders intensiven Betreuung, da sie sich selbst, meist infolge eines geringen Gesundheitsbewusstseins, vernachlässigen, in einem schlechten Ernährungszustand befinden und häufig in eine soziale Isolation geraten. Oft bemerken die Patientinnen ihre Schwangerschaft erst in einem fortgeschrittenen Stadium und begeben sich aus Angst vor öffentlichen Einrichtungen, die ihnen die Kindeserziehung verweigern könnten, erst sehr spät in medizinisch-therapeutische Betreuung [2]. Die Schwangerschaft zu verleugnen und sich nicht behandeln zu lassen ist mit einem hohen gesundheitlichen Risiko verbunden. Vor allem Untersuchungen aus den USA betonen die potenziell eintretenden Komplikationen, die der Drogenkonsum für die schwangere Frau, den Fetus und das Neugeborene mit sich bringen können [3,4]. Fetale Konsequenzen ergeben sich aber nicht nur durch den Konsum von illegalen Substanzen, sondern in einem noch größeren Ausmaß durch den Missbrauch und die Abhängigkeit von legalen Substanzen wie Alkohol und Nikotin, die bekann- 
terweise substanzbedingt eine Alkoholembryopathie bzw. ein fetales Tabaksyndrom auslösen können [5].

Demgegenüber führt anhaltender Heroinkonsum während der Schwangerschaft häufig zu Frühgeburten, niedrigem Geburtsgewicht und einem schlechten Ernährungszustand des Neugeborenen [6]. Die Mehrzahl der unter diesen Bedingungen geborenen Kinder leidet unter einem neonatalen Entzugssyndrom (NAS) [7].

Die internationale Standardtherapie der Opioidabhängigkeit während der Schwangerschaft ist die Erhaltungstherapie mit synthetischen Opioiden. Leider kommt dieser Therapiestandard in Europa nur eingeschränkt zur Anwendung, da in einigen Ländern Zentral- und Osteuropas nach wie vor keine Opioiderhaltungstherapie verfügbar ist [8]. Eine Einbindung der opioidabhängigen Schwangeren und ihres meist ebenfalls abhängigen Partners in ein multiprofessionelles Betreuungsprogramm optimiert die Versorgung der Schwangeren/Mutter und des Kindes. Regelmäßige fachärztliche Kontrollen der Mutter und des Kindes erhöhen die Sicherheit und bieten die Möglichkeit der interdisziplinären Abklärung und Behandlung zusätzlich auftretender gesundheitlicher Probleme.

\section{Orale Opioiderhaltungstherapie}

Opioidabhängige, die die Kriterien der Opioidabhängigkeit erfüllen, sollten mit einer Opioiderhaltungstherapie behandelt werden [9]. Eine anhaltende Abstinenz wäre zwar das ideale Ziel, das allerdings - wie durch die Forschung der letzten Jahre bewiesen - für die Mehrheit als unrealistisch anzusehen ist [10]. Es stehen im Sinne der Diversifikation der Opioiderhaltungstherapie verschiedene Medikamente zur Auswahl [11]. Methadon wird seit 1965 verwendet und ist die bislang am besten untersuchte Substanz [12]. In den letzten Jahren wurden aber auch retardierte Morphine und Buprenorphin zur Erhaltungstherapie verwendet und ihre Effektivität bestätigt [13]. Im Gegensatz zu Methadon und retardierten Morphinen, die reine my-Agonisten sind, besitzt Buprenorphin eine partiell my-agonistische Wirksamkeit und eine kappa-antagonistische Wirkung. Es wird bereits seit
Jahren zur Erhaltungstherapie verwendet [14,15]. Der pharmakologische Vorteil dieser Substanz ist sein geringeres physisches Suchtpotenzial, seine höhere Sicherheit bezüglich letaler Überdosierungen und sein geringeres Missbrauchspotenzial. Zudem treten bei Detoxifizierung Entzugssymptome in weit geringerem Ausmaß als bei herkömmlicherweise angewandten Opioiden auf [16]. Im Rahmen einer Erhaltungstherapie darf aber durch eine erhöhte Erwartungshaltung des Therapeuten keine Detoxifizierung forciert werden, da diese häufig zur Destabilisierung und Rückfällen mit illegalen Substanzen führt.

Nachdem Finnegan als eine der ersten Autorinnen die Anwendung von Methadon während der Schwangerschaft propagierte, folgten zahlreiche Untersuchungen, die diese Empfehlung unterstützten [17]. Wesentlich ist, dass Methadon während der Schwangerschaft aufgrund einer schwangerschaftsbedingten Enzyminduktion meist zweimal täglich verabreicht werden muss [18]. Eine Erhaltungstherapie mit Methadon verbessert die Schwangerschaftsvorsorge und ermöglicht gesunde Neugeborene [19].

Obwohl Methadon zu einer nachweislichen Stabilisierung gravider opioidabhängiger Patientinnen führt, findet es bedingt durch sein Nebenwirkungsprofil bei einem Teil der Patientinnen zu geringe Akzeptanz [20]. Daher kamen auch andere synthetische Opioide zur Anwendung. Dabei erwiesen sich retardierte Morphine, auch während der Schwangerschaft, als sichere und wirksame Alternative zu Methadon [21].

Die jüngsten Untersuchungen wurden mit sublingual $\mathrm{zu}$ applizierendem Buprenorphin unternommen [22,23]. Dabei zeigte sich, dass Kinder von mit Buprenorphin behandelten Müttern gesund sind und meist kein oder nur ein gering ausgeprägtes NAS (neonatales Abstinenzsyndrom) entwickelten. Diese ersten Ergebnisse sind richtungweisend, müssen jedoch aufgrund der geringen Zahl der Patientinnen mit Vorsicht interpretiert werden und bedürfen noch der Bestätigung durch weitere Forschungsarbeiten, ehe sie generell zur Entwicklung neuer Therapiestandards Anlass geben können.

\section{Schwangerschaft und Drogen}

Drogenambulanz,

Klinische Abteilung für Allgemeine Psychiatrie, Universitätsklinik für Psychiatrie, AKH Wien
Abb. 1 Multiprofessionelles Betreuungsmodell für gravide substanzabhängige Frauen.

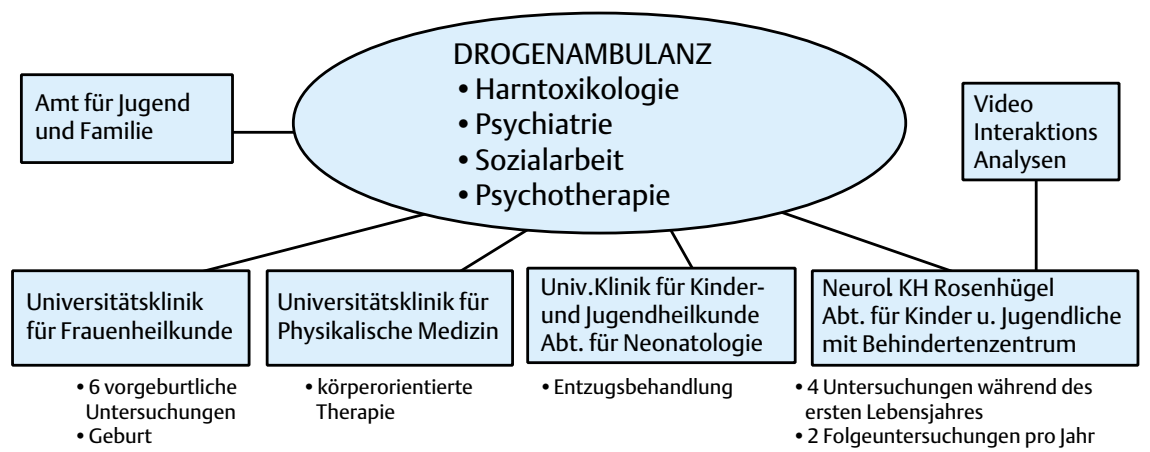




\section{Methodik}

An der Drogenambulanz der Universitätsklinik für Psychiatrie des Allgemeinen Krankenhauses Wien wurde 1994 ein standardisiertes Betreuungsprogramm für schwangere Opioidabhängige etabliert. Ein multiprofessionelles Team aus Psychiater(inne)n, Sozialarbeiter(inne)n und einer Psychotherapeutin gewährleistet in enger Zusammenarbeit mit Fachärzt(inn)en der Gynäkologie/Neonatologie eine optimale Versorgung und Betreuung der Patientinnen.

Das Ausmaß des vergangenen und aktuellen Drogenkonsums wird standardisiert mit der europäischen Version des „Addiction Severity Index“ (ASI) [24] und das Persönlichkeitsprofil nach dem TCI (Temperament and Character Inventory) [25] erhoben. Eine laborchemische Untersuchung erfolgt $\mathrm{zu}$ Beginn der Betreuung routinemäßig u.a. auf HIV und Hepatitis. Wöchentlich werden toxikologische Harnkontrollen auf Zusatzkonsum von Opiaten, Kokain und Benzodiazepinen und Cannabis mittels „Emit ${ }^{\circledR} “$ überprüft [26].

Die Patientinnen erhalten entweder Methadon, retardierte Morphine oder Buprenorphin. Methadon wird als orale Lösung (Razemat aus L- und R-Methadon), retardierte Morphine (Morphinhydrochlorid: Vendal $\operatorname{retard}^{\circledR}$ ) zweimal täglich als Tablette und Buprenorphin (Subutex ${ }^{\circledR}$ ) einmal täglich als Sublingualtablette verabreicht. Eine Mitgaberegelung der Medikamente besteht für alternierende Tage und für das Wochenende. Unabhängig von der Art der Opioiderhaltungstherapie erhalten alle Patientinnen zweimal wöchentlich eine psychosoziale und einmal wöchentlich eine psychotherapeutische Betreuung. Im Rahmen der psychotherapeutischen Gruppe, die gemeinsam für Schwangere und junge Mütter angeboten wird, wird neben der Rückfallprophylaxe und Verhaltensmodifizierung, Selbstbehauptungstraining und dem Erlernen neuer Copingstrategien auch die Mutterrolle thematisiert. Die Patientinnen erhalten dadurch die Gelegenheit, Veränderungsmöglichkeiten ihres Lebensstils zu erkennen, ihre Erkrankung zu akzeptieren und dadurch Außenprojektionen zu vermeiden. Regelmäßige gynäkologische (sechs präpartale Kontrollen) und neonatologische Untersuchungen finden als Teil des interdisziplinären Betreuungsprogrammes statt.

Für die vorliegende Untersuchung wurden alle graviden Patientinnen, die sich in einem Behandlungszeitraum von 42 Monaten in einer Erhaltungstherapie befanden, herangezogen. Es wurden 113 schwangere opioidabhängige (DSM IV: 304,0) oder polytoxikomane (DSM IV: 304,8) Frauen in die Untersuchung eingeschlossen [27].

Das Ziel unserer Untersuchung war die Analyse der Faktoren, die eine Stabilisierung schwangerer Opioidabhängiger beeinflussen, und die Evaluierung der Parameter, die einen Einfluss auf die Beibehaltung der Sorge für das Kind darstellen.

\section{Statistik}

Die statistische Analyse erfolgte mittels des Statistical Package for the Social Sciences (SPSS) [28]. Die Daten auf Intervallskalenniveau wurden anhand von Mittelwert, Standardabweichung und Variationsbreite (range) beschrieben. Die Mittelwertsvergleiche wurden nach Überprüfung der Voraussetzung der Normalverteilung der Daten parametrisch mittels
t-Test für unabhängige Stichproben bzw. einfacher Varianzanalyse durchgeführt, bei Nichtvorliegen der Normalverteilung nonparametrisch mit dem Mann-Whitney-U-Test bzw. dem Kruskal-Wallis-Test. Zur Darstellung der Unterschiede von Häufigkeitsangaben, in Bezug auf demografische Daten als auch bei den Ergebnissen der toxikologischen Harnkontrollen wurde der $\chi^{2}$-Test verwendet.

\section{Ergebnisse}

Von 113 opioidabhängigen schwangeren Patientinnen, die über einen Zeitraum von 42 Monaten in unserer Ambulanz behandelt wurden, wiesen 61 Frauen (54,1\%) beim Erstkontakt eine Opioidabhängigkeit (DSM IV: 304,0$)$ und 52 (45,9\%) eine Polysubstanzabhängigkeit (DSM IV: 304,8) auf.

Tab. 1 Diagnose und Schwangerschaftsergebnis $(n=113)$

\section{Diagnose:}

Opioidabhängigkeit

$61(54,1 \%)$

Polytoxikomanie

$52(45,9 \%)$

Schwangerschaftsergebnis:

Schwangerschaftsabbruch $\quad 7(6,2 \%)$

Abortus $4(3,5 \%)$

Totgeburt

$4(3,5 \%)$

Geburten

$94(83,2 \%)$

noch bestehende Schwangerschaft

$7(6,2 \%)$

Im Untersuchungszeitraum entschieden sich sieben Frauen vor der zwölften Schwangerschaftswoche für einen Schwangerschaftsabbruch, vier Frauen erlitten einen Abortus und eine Frau eine Totgeburt. Zum Untersuchungszeitpunkt waren noch sieben Frauen schwanger. Alle andere Frauen ( $\mathrm{n}=94$ ) brachten gesunde Kinder zur Welt.

Zum Evaluationszeitpunkt lebten 56 Kinder $(59,6 \%)$ bei ihren Müttern, während sich 38 (40,4\%) Kinder in der Fürsorge anderer Personen befanden.

Tab. 2 Verbleib der Kinder $(\mathrm{n}=94)$

Kinder verbleiben bei ihrer Mutter

$56(59,6 \%)$

Kinder verbleiben nicht bei ihrer Mutter

$38(40,4 \%)$

Zeitpunkt der Kindesabnahme $(n=38)$ :

sofort nach der Geburt

$22(57,8 \%)$

während des Untersuchungszeitraumes

$16(42,2 \%)$

Verbleib der sofort nach der Geburt

fremduntergebrachten Kinder $(n=22)$ :

Adoption

$5(22,7 \%)$

Pflegeeltern

$7(31,8 \%)$

Familienangehörige

$10(45,5 \%)$

Verbleib der während des Untersuchungszeitraumes

fremduntergebrachten Kinder $(n=16)$

Pflegeeltern

$9(56,3 \%)$

Familienangehörige

$7(43,7 \%)$

Für 22 der fremduntergebrachten Kinder veranlasste das Jugendamt sofort nach der Geburt ein Übergabeverbot an die Mutter, da es nicht gelang, diese Frauen zu stabilisieren, und 
die Schwere ihrer Suchterkrankung eine Selbstversorgung des Kindes nicht zuließ. Sieben dieser Kinder wurden bei Pflegefamilien in Fürsorge gegeben. Zehn Mütter waren mit der Entscheidung des Jugendamtes, das Sorgerecht auf die Großeltern zu übertragen, einverstanden. Eine Entscheidung, die dadurch erleichtert wurde, da sich die Mütter meist in einem gemeinsamen Haushalt mit den Großeltern befanden und so weiterhin mit ihrem Kind zusammenleben konnten. Fünf Kinder wurden auf Wunsch der Mutter nach der Geburt zur Adoption freigegeben. Als Grund für die Adoption gaben 2 Frauen an, dass die Schwangerschaft im Zuge einer Vergewaltigung entstanden sei. Eine Frau erkannte, dass sie infolge ihrer schweren Suchterkrankung unmöglich für ein Kind sorgen könne, und zwei Frauen lehnten das Kind aufgrund der Trennung vom Kindsvater ab. Bei diesen fünf Frauen stand die Adoption bereits in der ersten Woche der Behandlung fest und dennoch verhielten sich alle während des Betreuungszeitraumes der Schwangerschaft zufrieden stellend kooperativ.

Von den 16 Kindern, die während des Untersuchungszeitraumes den Müttern abgenommen wurden, wurde in 7 Fällen die elterliche Sorge auf Familienangehörige, meist Großeltern, und in 9 Fällen auf Pflegefamilien übertragen.

In den demografischen Daten finden sich in den zwei Gruppen keine wesentlichen Unterschiede. Eindrucksvoll ist jedoch, dass mit Ausnahme von 8 Patientinnen, die keine Ausbildung besitzen, alle untersuchten Frauen ein ausreichendes Bildungsniveau aufweisen. Weiter zeigte sich, dass über die Hälfte der Frauen (59\%) einen ebenfalls opioidabhängigen Partner haben (Tab. 3).

Der Vergleich der medikamentösen Therapie beim Erstkontakt bringt zwischen den Frauen, die mit ihrem Kind leben $(n=54)$, und jenen, die ihre Kinder in andere Pflege $(n=38)$ geben mussten, keine signifikanten Unterschiede. 50 (54\%) der untersuchten Frauen befanden sich bereits zum Zeitpunkt des Erstkontaktes in einer Opioiderhaltungstherapie (Tab. 4).

Frauen, die ihre Kinder bei sich behalten konnten, hatten zum Geburtszeitpunkt annähernd doppelt so lange eine Opioiderhaltungstherapie $(p=0,021)$. Zudem zeigt sich, dass Frauen eher das Sorgerecht für ihre Kinder bekommen, wenn sie sich früh in eine stabilisierende Betreuung begeben $(p=0,034)$. In der Gruppe der mit Methadon behandelten Schwangeren korreliert die Wegnahme der Kinder mit der Höhe der Methadondosis $(p=0,015)$. Frauen, die ihre Kinder nicht übergeben bekamen, weisen während der Schwangerschaft häufiger einen Zusatzkonsum von Opiaten und Benzodiazepinen auf $(\mathrm{p}<0,05)$ (Tab. 5).

Ein Hepatitis-C-positiver Befund wird für 71 Patientinnen (76\%) und HIV für vier Patientinnen (4\%) laborchemisch nachgewiesen. Alle Neugeborenen zeigten einen negativen Infektionsstatus.

Die Teilnahme an der wöchentlichen Psychotherapiegruppe zeigte ebenfalls einen wesentlichen Einfluss, denn Patientinnen, die für ihre Kinder sorgen konnten, nahmen häufiger an der therapeutischen Gruppe teil $(p=0,03)$.

\begin{tabular}{|c|c|c|c|}
\hline & $\begin{array}{l}\text { Kind lebt bei } \\
\text { seiner Mutter } \\
n=56(59,6 \%)\end{array}$ & $\begin{array}{l}\text { Kind lebt nicht bei } \\
\text { seiner Mutter } \\
n=38(40,4 \%)\end{array}$ & $\mathrm{p}$ \\
\hline $\begin{array}{l}\text { Familienstatus: } \\
\text { ledig } \\
\text { verheiratet } \\
\text { geschieden } \\
\text { verwitwet }\end{array}$ & $\begin{array}{r}26(46,5 \%) \\
13(23,2 \%) \\
15(26,7 \%) \\
2(3,6 \%)\end{array}$ & $\begin{array}{r}19(50,0 \%) \\
12(31,5 \%) \\
7(18,5 \%) \\
0(\quad 0 \%)\end{array}$ & n.s. \\
\hline $\begin{array}{l}\text { Ausbildung: } \\
\text { keine } \\
\text { Hauptschule } \\
\text { Berufschule } \\
\text { Hochschule }\end{array}$ & $\begin{array}{r}4(7,2 \%) \\
31(55,4 \%) \\
9(16,0 \%) \\
12(21,4 \%)\end{array}$ & $\begin{array}{r}4(10,5 \%) \\
16(42,1 \%) \\
13(34,2 \%) \\
5(13,2 \%)\end{array}$ & n.s. \\
\hline $\begin{array}{l}\text { Partner: } \\
\text { opioidabhängiger Partner } \\
\text { nicht abhängiger Partner }\end{array}$ & $\begin{array}{l}34(60,7 \%) \\
22(39,3 \%)\end{array}$ & $\begin{array}{l}21(55,3 \%) \\
17(44,7 \%)\end{array}$ & n.s. \\
\hline $\begin{array}{l}\text { Opioiderhaltungstherapie } \\
\text { bei der Geburt: } \\
\text { Methadon } \\
\text { retardierte Morphine } \\
\text { Buprenorphin }\end{array}$ & $\begin{array}{l}33(58,9 \%) \\
12(21,4 \%) \\
11(19,7 \%)\end{array}$ & $\begin{array}{l}17(44,4 \%) \\
21(55,6 \%) \\
-\end{array}$ & 0,0001 \\
\hline $\begin{array}{l}\text { Geschlecht des Kindes: } \\
\text { männlich } \\
\text { weiblich }\end{array}$ & $\begin{array}{l}35(62,5 \%) \\
21(37,5 \%)\end{array}$ & $\begin{array}{l}20(52,6 \%) \\
18(47,4 \%)\end{array}$ & n.s. \\
\hline $\begin{array}{l}\text { Zahl der Geburten: } \\
\text { erstes Kind } \\
\text { zweites Kind } \\
\text { drittes Kind } \\
\text { viertes Kind }\end{array}$ & $\begin{array}{r}36(64,3 \%) \\
12(21,4 \%) \\
6(10,7 \%) \\
2(3,6 \%)\end{array}$ & $\begin{array}{r}22(57,9 \%) \\
12(31,6 \%) \\
3(7,9 \%) \\
1(2,6 \%)\end{array}$ & n.s. \\
\hline
\end{tabular}

Tab. 3 Vergleich der demografischen Daten der Mütter, die mit $(n=56)$ und ohne $(n=38)$ ihr Kind leben 


\begin{tabular}{|c|c|c|c|}
\hline & $\begin{array}{l}\text { Kind lebt bei } \\
\text { Mutter } \\
n=56\end{array}$ & $\begin{array}{l}\text { Kind lebt } \\
\text { nicht bei Mutter } \\
\mathrm{n}=38\end{array}$ & $\mathrm{p}$ \\
\hline $\begin{array}{l}\text { Gravide ohne Erhaltungstherapie bei } \\
\text { Erstkontakt }(n=44)\end{array}$ & $20(35,7 \%)$ & $24(63,2 \%)$ & \\
\hline $\begin{array}{l}\text { Gravide in einer Erhaltungstherapie beim } \\
\text { Erstkontakt }(n=50)\end{array}$ & $36(64,3 \%)$ & $14(36,8 \%)$ & n.s. \\
\hline \multicolumn{4}{|l|}{$\begin{array}{l}\text { Substanzen der Erhaltungstherapie beim } \\
\text { Erstkontakt: }\end{array}$} \\
\hline $\begin{array}{l}\text { Methadon } \\
\text { retardierte Morphine }\end{array}$ & $\begin{array}{r}32(88,8 \%) \\
4(11,2 \%)\end{array}$ & $\begin{array}{r}13(92,8 \%) \\
1(7,2 \%)\end{array}$ & n.s. \\
\hline
\end{tabular}

Tab. 4 Opioiderhaltungstherapie zum Erstkontakt

\section{Diskussion}

Da opioidabhängige Schwangere eine Risikopopulation darstellen, gilt es, möglichst rasch ein stabilisiertes Zustandsbild der werdenden Mutter zu erzeugen, um den Neugeborenen die bestmögliche Ausgangsbedingung zu schaffen. In dieser Untersuchung wurden Faktoren analysiert, die eine Stabilisierung der Frauen während der Schwangerschaft und somit auch ihre Erziehungsfähigkeit, beeinflussen.

Die Mehrzahl (60\%) der untersuchten Frauen konnte für ihre Kinder selbst sorgen. Je früher sich eine Patientin in Betreuung begab und je länger sie sich in einer Opioiderhaltungstherapie befand, desto eher konnte sie ihr Kind behalten. Obwohl einige Kritiker den Gebrauch von oralen synthetischen Opioiden historisch als „Ersatzsucht“ bezeichnen [29], haben zahlreiche Untersuchungen die Effektivität dieser Medikamente belegt.

Opioidabhängigkeit definiert sich als eine der schwersten chronischen psychiatrischen Erkrankungen und das Vertreten der Ideologie einer Einmalintervention mit nachfolgender Heilung würde für ein Nicht-Akzeptieren des heutigen wissenschaftlichen Standards sprechen [30]. Gerade für heroinabhängige Schwangere dient diese Form der Behandlung auch zur Absicherung der entsprechenden präpartalen Betreuung. Darüber hinaus behindern unrealistische Erwartungen hinsichtlich einer Heilung der Abhängigkeit, gerade in einer so sensiblen Phase wie der Gravidität, die Effektivität der Behandlung und sollten durch eine entsprechende Aufklärung behoben werden [31]. In diesem Zusammenhang ist auch vor einer zu raschen Detoxifizierung während der Schwangerschaft zu warnen, da diese ein fetales Stresssyn- drom und dadurch eine Frühgeburt erzeugen sowie durch Rückfälle zu einer neuerlichen Destabilisierung führen kann. Die Erwartung, zum Zeitpunkt der Geburt frei von Opioiden sein zu müssen, gilt heute als überholt. Man weiß, dass gerade während der Schwangerschaft zur Reduktion des fetalen und mütterlichen Risikos eine stabile Dosierung unverzichtbar ist und dass im letzten Trimenon aufgrund einer schwangerschaftsbedingten Enzyminduktion eine Dosiserhöhung notwendig werden kann [32].

Schwangerschaft kann auch für nicht abhängige Frauen eine psychische Belastung darstellen und führt gerade auch in der Zeit nach der Geburt gehäuft zu depressiven Reaktionen (Post-partum-Depression) [33]. Für substanzabhängige Mütter gestaltet sich die Situation nach der Geburt um vieles schwieriger. Wesentlich ist daher, die kontinuierliche Betreuung auch postpartal fortzusetzen. Die Opioiderhaltungstherapie scheint ein guter Indikator für die Stabilisierung der Patientinnen zu sein und auch die Diversifikation der medikamentösen psychopharmakologischen Therapie könnte in $\mathrm{Zu}$ kunft zu einer verbesserten Versorgung dieser Population führen, indem sie die Compliance erhöht, wenn zum Beispiel Methadon durch auftretende Nebenwirkungen teilweise nur geringe Akzeptanz findet.

Unser Untersuchungsergebnis zeigt den wesentlichen Einfluss einer frühzeitigen und regelmäßigen therapeutischen Intervention. Gerade jene Frauen, die nicht die elterliche Sorge für ihre Kinder erhielten, brachen aufgrund einer geringeren Compliance das strukturierte Behandlungsprogramm vorzeitig ab und konnten dadurch das medizinische und psychosoziale Betreuungsangebot nicht nutzen. Der Erstkontakt dieser Frauen fand erst später - durchschnittlich in

Tab. 5 Opioiderhaltungstherapie zum Zeitpunkt der Geburt

\begin{tabular}{|c|c|c|c|c|c|c|c|}
\hline & \multicolumn{3}{|c|}{ Mütter mit Kind $(n=56)$} & \multicolumn{4}{|c|}{ Mütter ohne Kind $(n=38)$} \\
\hline & Mean & SD & Range & Mean & SD & Range & $\mathrm{p}$ \\
\hline Alter (in Jahren) & 26,02 & 5,03 & $18-40$ & 24,85 & 5,04 & $16-37$ & n.s. \\
\hline Schwangerschaftswoche bei Erstkontakt & 17,98 & 6,87 & $6-36$ & 21,79 & 7,97 & $12-39$ & 0,034 \\
\hline Dauer der Opioiderhaltungstherapie (in Monaten) & 8,79 & 12,34 & $1-73$ & 4,37 & 4,75 & $1-32$ & 0,021 \\
\hline Methadondosis (in mg) & 34,97 & 32,04 & $10-120$ & 61,86 & 27,03 & $30-145$ & 0,015 \\
\hline retardierte Morphindosis (in mg) & 300,14 & 111,87 & $120-400$ & 252,40 & 152,97 & $10-500$ & n.s. \\
\hline Buprenorphindosis (in mg) & 7,84 & 4,12 & $2-10$ & - & - & - & - \\
\hline Zeitpunkt der Kindesabnahme (Monate nach der Geburt) & - & - & - & 4,17 & 7,24 & $0-28$ & - \\
\hline
\end{tabular}


der 22. Schwangerschaftswoche - statt, während die übrigen Schwangeren bereits in der 18. Schwangerschaftswoche medizinische Betreuung suchten. Demnach war auch der Untersuchungszeitraum, um eine stabilisierende Betreuung $\mathrm{zu}$ gewährleisten, kürzer. Tatsächlich stellt die späte Inanspruchnahme einer stabilisierenden Betreuung und die schlechte Compliance während der Behandlung eines der größten Probleme in der Versorgung dieser Risikopopulation dar. Durch eine entsprechende Aufklärung und Etablierung von Standards kann die Angst abhängiger schwangerer Frauen auch vor öffentlichen Einrichtungen verringert werden [31]. Mit dem Ziel, gravide substanzabhängige Frauen bereits im ersten Trimenon zu behandeln, wäre es wesentlich, auch Standards in öffentlichen Einrichtungen (z.B. Jugendämter) einzubeziehen. Schon alleine durch 60000 mit Buprenorphin behandelten Patientinnen in Frankreich wird sich auch die Zahl der Konzeptionen unter dieser Substanz erhöhen, was eine Aufklärung zur Erkennung der Gravidität und eine frühzeitige Behandlungsinitiierung nahe legt.

Gleichermaßen wie sich eine suffiziente pharmakologische Behandlung und psychosoziale Betreuung positiv auf das Verbleiben des Kindes bei der Mutter auswirkten, konnte auch der signifikant positive Einfluss der psychotherapeutischen Gruppe evaluiert werden. Als positiv erlebten die Teilnehmerinnen dabei das Prinzip einer gemischten Gruppe, die sowohl aus Graviden als auch aus jungen Müttern bestand.

Zusätzlich erschwert sicherlich die hohe psychiatrische Komorbidität substanzabhängiger Patientinnen ihre Behandlung und sollte daher vor allem von erfahrenen Psychiater(inne)n im Rahmen eines multiprofessionellen Therapieprogramms vorgenommen werden [34]. Während niedergelassene Ärzte/ Ärztinnen mit entsprechendem Ausbildungshintergrund durchaus kompetent in der Versorgung Substanzabhängiger sein können, zeigt sich dies bei dieser Risikogruppe als primär nicht empfehlenswert, da nur durch ein multidisziplinäres System in Spezialkliniken - schon alleine aufgrund der geografischen Nähe diverser Abteilungen - eine Erleichterung in der Compliance der Untersuchungen gegeben ist.

Abgeleitet von unserem Ergebnis ergibt sich die Empfehlung, gravide substanzabhängige Patientinnen in einem Schwerpunktzentrum mit interdisziplinärer Betreuung (Sozialarbeit, Psychiatrie, Psychotherapie) zu behandeln, wo gleichermaßen eine engmaschige Vernetzung mit gynäkologisch und neonatologischen Fachrichtungen besteht. Außerdem sollte eine professionelle Weiterbehandlung der Mütter und die Verfolgung des Entwicklungsverlaufs der Kinder einen wesentlichen Beitrag zur Primärprävention darstellen. Für die Effektivität des Programms spricht auch, dass selbst Frauen, die wussten, dass sie ihr Kind zur Adoption freigeben werden, die Behandlung nicht vorzeitig beendeten.

\section{Danksagung}

Wir danken unseren Kooperationspartnern an der Gynäkologie (Martin Langer, Christian Schatten) und Neonatologie (Manfred Weninger) im AKH, als auch des Neurologischen Krankenhauses Rosenhügel (Ernst Berger, Thomas Elstner) sowie dem Team der Drogenambulanz für ihre Mitarbeit, die diese Untersuchung erst ermöglicht haben.

\section{Literatur}

${ }^{1}$ Fischer G. Treatment of opioid dependence in pregnant women. Addict 2000; 8: 1141-1144

2 Deleon G, Jainchill N. Residential therapeutic communities for female substance abusers. Bull NY Acad Med 1991; 67: 277-290

${ }^{3}$ Finnegan LP. Clinical effects of pharmacologic agents on pregnancy, the fetus and the neonate. Ann N Y Acad Sci 1976; 281 : $74-89$

${ }^{4}$ Chasnoff IJ. Drugs, alcohol, and the neonate: pay now or pay later. JAMA 1991; 266: 1567-1568

${ }^{5}$ Fingerhut LA, Kleinmann JC, Kendrick JS. Smoking before, during and after pregnancy. Am J Public Health 1990; 80: 541-544

${ }^{6}$ Zuckermann B, Brown E. Maternal substance abuse and infant development. In: Tsang R (Hrsg). Handbook of Infant Mental Health. New York: Guilford Press, 1993: 143-158

${ }^{7}$ Levy M, Spino M. Neonatal withdrawal syndrom: Associated drugs and pharmacological managment. Pharmacother 1993; 13: $202-211$

${ }^{8}$ European Monitoring Center For Drugs And Drug Addiction. Reviewing current practice in drug substitution treatment in the European Union. E.M.C.D.D.A Report 2000

${ }^{9}$ Schneider C, Fischer G, Diamant K et al. Schwangerschaft und Drogenabhängigkeit. Wien Klin Wochenschr 1996; 108/19: 611-614

${ }^{10}$ Hser YI, Hoffman V, Grella CE, Anglin D. A 33-year follow-up of narcotic addicts. Arch Gen Psychiatry 2001; 58: 505-508

${ }^{11}$ Fischer G, Eder H, Jagsch R et al. Relapse prevention with opioid agonists and antagonists in heroin addiction. Europ Neuropsychopharmacol 1997; 7: 99

12 Dole VP, Nyswander M. A medical treatment for diacethylmorphine (heroin) addiction. JAMA 1965; 193: 80-84

${ }^{13}$ Fischer G, Presslich O, Diamant K et al. Oral morphine-sulphate in treatment of opiate dependent patients. Alcohol 1996; 32: $35-43$

${ }^{14}$ Johnson RE, Jaffe JH, Fudala PJ. A controlled trial of buprenorphine treatment for opioid dependence. J Am Med Assoc 1992; 267: 2750-2755

${ }^{15}$ Fischer G, Gombas W, Eder H et al. Vergleichsuntersuchung von Buprenorphin und Methadon im Rahmen der Erhaltungstherapie Opiatkranker. Nervenarzt 1999; 70: 795-802

${ }^{16}$ Diamant K, Fischer G, Schneider C et al. Outpatient opiate detoxification treatment with buprenorphine. Preliminary investigation. Eur Addict Res 1998; 4: 198-202

${ }^{17}$ Finnegan LP. Management of pregnant drug-dependent women. Ann N Y Acad Sci 1978; 311: 135-146

${ }^{18}$ Jarvis MA, Wu-Pong S, Kniseley JS, Schnoll SH. Alterations in methadone metabolism during late pregnancy. J Addict Dis 1999; 18: 51-61

${ }^{19}$ Blinick G, Jerez E, Wallach RC. Methadone maintenance, pregnancy and progeny. JAMA 1973; 225: 477-479

${ }^{20}$ Langrod J, Lowinson J, Ruiz P. Methadone treatment and physical complaints: a clinical analysis. Int J Addict 1981; 16: 947-952

${ }^{21}$ Fischer G, Jagsch R, Eder $\mathrm{H}$ et al. Comparison of methadone and slow-release morphine maintenance in pregnant addicts. Addict 1999; 94: 231-239

${ }^{22}$ Reisinger M. Treatment of four pregnant heroin addicts with buprenorphine. History and outcome, College on Problems of Drug Dependence. NIDA Research Monograph Series 1995; 261

${ }^{23}$ Fischer G, Johnson RE, Eder H et al. Treatment of opioid-dependent pregnant women with buprenorphine. Addict 2000; 95: 239-244

${ }^{24}$ Mclellan A, Kushner H, Metzger D et al. Addiction severity index. 5th edn. J Subst Abuse Treat 1992; 9: 199-213 
${ }^{25}$ Cloninger CR, Svrakic DM, Przybeck TR. A Psychobiological Model of Temperament and Character. Arch Gen Psychiatry 1993; 50: 975-990

${ }^{26}$ Emit Assay Troubleshooting Guide. Palo Alto: Syva Co 1983

${ }^{27}$ American Psychiatric Association. Diagnostical and statistical manual and mental disorders. 4th edn. (DSM-IV). Washington D.C, 1994

${ }^{28}$ Bühl A, Zöfel P. SPSS für Windows Version 7.5. Praxisorientierte Einführung in die moderne Datenanalyse. Addison-WesleyLongman. Bonn, 1998

${ }^{29}$ Sass H. Pro und Contra Substitution Drogenabhängiger mit Methadon. Nervenarzt 1991; 62: 521-523

${ }^{30}$ Edwards G. Addiction treatment and the making of large claims. Addict 2000; 95: 1755-1757

${ }^{31}$ Hagan TA, Finnegan LP, Nelson-Zlupko L. Impediments to comprehensive treatment models for substance-dependent women: treatment and research questions. J Psychoact Drug 1994; 26: $163-171$

${ }^{32}$ Pond SM, Kreek MJ, Tong TG et al. Altered methadone pharmacokinetics in methadone-maintained pregnant women. J Pharm Exp Ther 1985; 233: 1-6

33 Brockington IF, Cernik KF, Schofield EM et al. Puerperal Psychosis. Arch Gen Psychiatry 1981; 38: 829-833

34 Woods JR. Clinical management of drug dependency in pregnancy. NIDA Research Monograph Series 1995; 149: 39-57
Univ.-Prof. Dr. Gabriele Fischer

Drogenambulanz

Klinische Abteilung für Allgemeine Psychiatrie,

Universitätsklinik für Psychiatrie

AKH Wien

Währinger Gürtel 18-20

1090 Wien

Österreich

E-mail: gabriele.fischer@akh-wien.ac.at 